\title{
Relación entre Cognición Social y Funciones Ejecutivas en Niños con Trastorno Negativista Desafiante
}

\author{
Relationship between Social Cognition and Executive Functions in Children with \\ Oppositional Defiant Disorder
}

\author{
Juan Carlos Restrepo Botero ${ }^{1}$, Claudia Marcela Arana Medina ${ }^{2}$, Alexander Alvis Rizzo ${ }^{3}$, Ana \\ Catalina Gómez Aristizabal ${ }^{4}$ y Elizabeth Hoyos Zuluaga ${ }^{5}$
}

\section{Resumen}

En el presente estudio se explora la relación entre cognición social y funciones ejecutivas en nueve niños con trastorno negativista desafiante (TND) entre los 7 y 11 años (ciudad de Medellín, Colombia). Se evaluaron las funciones ejecutivas a través del Test de ejecución auditiva continua, el Test de la Figura Compleja de Rey Osterrieth, el Test de símbolos y dígitos, el Test de Clasificación de tarjetas de Wisconsin, el Test de rastreo, el Test de asociación controlada de palabras, la Curva de memoria verbal y el Test de Colores y Palabras de Stroop. La exploración de la cognición social se hizo a través del Test de la Mirada y la adaptación del Interpersonal Reactivity Index al español. Entre los resultados obtenidos se encuentran relaciones estadísticamente significativas entre Teoría de la mente-aprendizaje y empatía-control inhibitorio, organización/planeación visoespacial, secuenciación, conciencia fonológica, y estrategias ejecutivas de memoria.

Palabras clave: trastorno negativista desafiante, cognición social, funciones ejecutivas, empatía, teoría de la mente

\begin{abstract}
In the present study the relationship between social cognition and executive functions in nine children with oppositional defiant disorder (ODD) between 7 and 11 years of Medellin (Colombia) is explored. Executive functions were evaluated through the Continuous performance test, the Rey-Osterrieth Complex Figure Test, the Symbol Digit Substituting Test, the Wisconsin Card Sorting Test, the Trail Making Test, the Controlled Oral Word Association Test, the Memory curve and The Stroop Color and Word Test. The exploration of social cognition was made through The 'Reading the mind in the eyes' test revised version and Spanish adaptation of the Interpersonal Reactivity Index. Results highlighting the statistically significant relationship between theory of mind-learning and empathy-inhibitory control, organization/visuospatial planning, sequencing, phonological awareness, memory and executive strategies.
\end{abstract}

Keywords: oppositional defiant disorder, social cognition, executive functions, empathy, theory of mind

\footnotetext{
Artículo derivado de una investigación en la que se está evaluando la efectividad de un programa de rehabilitación neuropsicológico y psicosocial de niños entre los 7 y 12 años con TND durante 3 años financiada por la Fundación Universitaria Luis Amigó y la Corporación Universitaria Lasallista en Medellín, Colombia.

${ }^{1}$ Psicólogo, Especialista en Rehabilitación Neurocognitiva y Doctor en Psicología con orientación en Neurociencia Cognitiva Aplicada. Docente y Director del Grupo de Investigación en Psicología Aplicada, Corporación Universitaria Lasallista, Medellín, Colombia. Tel. (54)3007815692.

${ }^{2}$ Psicóloga, Especialista en terapia cognitiva y en psicología organizacional, Magister en Neuropsicología y PhD en Psicología con Orientación en Neurociencia Cognitiva Aplicada. Docente de la Fundación Universitaria Luis Amigó, Grupo de investigación: Neurociencias básicas y aplicadas. Línea de Investigación Perfiles Neurocognitivos y Psicología, Medellín-Colombia. Tel. (54)3006108847.

${ }^{3}$ Psicólogo y Magíster en Educación y Desarrollo Humano. Docente Programa de Psicología, Fundación Universitaria Luis Amigó, Grupo de investigación: Psicología Social y Salud Mental. Línea: Psicología Social y Salud Mental, Medellín-Colombia. Tel. (54)3103944033.

${ }^{4}$ Psicóloga, Especialista en Docencia Universitaria, Docente investigadora de la Fundación Universitaria Luis Amigó. Medellín- Colombia. Tel. (54)3006866165.

${ }^{5}$ Estadística y epidemióloga. Docente de la Universidad de San Buenaventura en Medellín, Colombia. Tel. (54)3127829716.

Correspondencia: Juan Carlos Restrepo Botero, e-mail: juarestrepo@lasallistadocentes.edu.co

Revista Iberoamericana de Diagnóstico y Evaluación - e Avaliação Psicológica. RIDEP · No42 · Vol.2 · 49-58 2016
} 


\section{Introducción}

Si bien la agresividad es un mecanismo de defensa y de adaptación inherente al ser humano, se comienza a considerar problemática cuando interfiere con la calidad de vida de la propia persona y su entorno. En los últimos años los trastornos de conducta diagnosticados han triplicado los reportados en los años noventa (Rabadán \& Giménez, 2012). Entre estas problemáticas se encuentra el trastorno negativista desafiante (TND). Aunque no es nuevo en el contexto escolar, tiene una prevalencia entre el 2 al $16 \%$ en la población, cuya frecuencia mayor se encuentra entre niños de 8-10 años y adolescentes de 13-16 años (Pino \& García, 2007). En el futuro este trastorno puede desembocar en conductas antisociales (Pardini \& Fite, 2010; Robles \& Romero, 2011).

El DSM IV-TR (American Psychiatric Association, 2003) lo define como un patrón recurrente (mínimo 6 meses) de comportamiento negativista, desafiante, desobediente y hostil, dirigido a las figuras de autoridad. Por su parte, el DSM-5 (American Psychiatric Association, 2013) hace un ajuste en los criterios diagnósticos identificando patrones de enfado/irritabilidad, discusiones/actitud desafiante o vengativa que dura por lo menos seis meses. Si bien los criterios diagnósticos para este trastorno siguen siendo un motivo de constante debate (Barry, Golmaryami, Rivera-Hudson, \& Frick, 2013; Kolko \& Pardini, 2010;), la literatura científica aporta características importantes que se identifican en estos niños y adolescentes, que pueden ayudar a definir cada vez mejor esta problemática.

Entre las características reportadas, se encuentran: dificultades desde la codificación hasta la generación e implementación de soluciones en tareas de solución de problemas y seguridad al generar respuestas agresivas (Coy, Speltz, DeKlyen, \& Jones, 2001; Matthys, \& Cuperus, 1999); fallas en la definición de situaciones problemas (Ison, 2004); conductas interferentes en el aula de clase mientras el docente proporciona sus explicaciones (Pino, \& García, 2007); falta de flexibilidad de pensamiento (Waschbusch, Walsh, Andrade, King, \& Carrey, 2007); dificultad en la autorregulación de sus propias emociones y fracaso para desarrollar conductas alternativas a la agresión (Ison-Zintilini, \& Morelato, 2008; Masi et al., 2014); manifestaciones diferenciales por sexo (de Ancos, \& Ascaso, 2011; Ezpeleta, De la Osa, Granero, \& Trepat, 2014); pérdida de procesos de aprendizaje social, deficiencias en inhibición y atención (Matthys, Vanderschuren, Schutter, \& Lochman, 2012); falta de culpa, pérdida de la empatía, expresión emocional deficiente, menor propensión a tener un impacto emocional por la angustia de los demás (Barry et al.,2013), entre otras.

Las anteriores dificultades en estos niños y adolescentes se asocian con otras problemáticas tales como: bajo rendimiento académico y deserción escolar (Corsi, Barrera, Flores, Perivancich, \& Guerra, 2009; Sliminng, Barrera, Flores, Perivancich, \& Guerra, 2009); tabaquismo, consumo de marihuana y drogas duras (Flory, Milich, Lynam, Leukefeld, \& Clayton, 2003; van Lier, Huizink, \& Vuijk, 2011), trastorno de ansiedad (Forehand, Jones, \& Parent, 2013), entre otras, que generan una carga familiar importante (Ezpeleta, et al., 2014).

Rabadán y Giménez (2012) han clasificado los factores de riesgo del TND en genéticos/individuales, familiares, ambientales y escolares. Entre los familiares, Roa y Del Barrio (2003) encuentran que los niveles altos de neuroticismo materno incrementan tales conductas en estos niños. Por su parte, Guaita (2007) reconoce el riesgo que genera la pobreza extrema.

Matthys et al. (2012) hacen un estudio detallado de las estructuras y zonas cerebrales alteradas en este trastorno. Se trata de la materia gris de los lóbulos temporales incluyendo la amígdala, la corteza prefrontal, la corteza orbitofrontal, el núcleo caudado. Estos compromisos afectan, entre otros procesos cognitivos, las funciones ejecutivas y la cognición social.

Las funciones ejecutivas son habilidades de orden superior necesarias para resolver problemas complejos. Entre estas se han identificado: la abstracción, anticipación, secuenciación, inhibición, planeación, categorización, jerarquización, conceptualización, razonamiento, toma de decisiones, flexibilidad de pensamiento, juicio social, capacidad de responder adaptativamente a estímulos emocionales, regulación y verificación de la acción, entre otras. Por lo tanto, involucra aspectos cognitivos, conductuales y emocionales (Restrepo, \& Molina, 2011).

La cognición social es un proceso cognitivo por medio del cual se puede interpretar, predecir, percibir adecuadamente los signos sociales y responderles de una manera adecuada. Incluye percepción, atribución, predicción de conductas emocionales, empatía, conocimiento de reglas sociales; detección de la mirada; entre otros. Román et al. (2012) identifican cuatro 
componentes principales: a) procesamiento emocional, b) teoría de la mente o la capacidad para representar las intenciones y creencias de las otras personas, c) percepción social y d) estilo o sesgo atribucional.

Diversos autores han identificado un compromiso de los niños y adolescentes con TND en las funciones ejecutivas (Ison, 2004; McKinney \& Morse, 2012; Matthys, et al., 2012) y la cognición social (Coy, et al., 2001; Muñoz, Carreras, \& Braza, 2004; Ison-Zintilini, \& Morelato, 2008).

El objetivo del presente estudio es explorar la relación entre las funciones ejecutivas y la cognición social de niños con TND entre los 7-11 años. Forman parte de un proyecto de investigación longitudinal en el que se está evaluando la efectividad de un programa de rehabilitación neuropsicológico y psicosocial implementado durante 3 años.

\section{Método}

\section{Diseño}

El diseño del presente estudio es de tipo no experimental, transversal y de nivel de alcance correlacional $^{1}$.

\section{Participantes}

Los participantes del presente estudio fueron estudiantes que cumplieran los siguientes criterios de inclusión: estar escolarizados, tener un diagnóstico de TND según los criterios del DSMIV-TR (American Psychiatric Association, 2003), tener entre 7-11 años, y un cociente intelectual (C.I.) mayor de 70. Nueve estudiantes de un colegio privado de la ciudad de Medellín (Colombia) cumplieron con los anteriores criterios y se incluyeron en el presente estudio.

\section{Instrumentos}

La inteligencia fue evaluada a través del Test breve de inteligencia de Kaufman (K-BIT, Kaufman, \& Kaufman, 1997).

Para la evaluación de las funciones ejecutivas se utilizaron: el Test de Ejecución Auditiva Continua (EAC; Lezak, 1995), el Test de la Figura Compleja de Rey Osterrieth (FCR; Rey, 2003), el Test de Símbolos y Dígitos (SDMT; Smith, 2002), el Test de Clasificación de Tarjetas de Wisconsin

\footnotetext{
${ }^{1}$ Se debe considerar que este artículo forma parte de la divulgación de resultados preliminares de una investigación longitudinal (de tres años) en la que se está sometiendo a prueba un programa de rehabilitación neuropsicológica y psicosocial de niños con TND.
}

(WCS; Heaton, Chelune, Talley, Kay, \& Curtiss, 2001), el Test de Rastreo (TDMT; Reitan, \& Wolfson, 1993), el Test de Asociación Controlada de Palabras (Lezak, Howieson, \& Loring, 2004), la Curva de Memoria Verbal (Ardila, \& Rosselli, 1992; Lezak, 1995) y el Test de Colores y Palabras de Stroop (Golden, 2001).

La exploración de la cognición social se hizo a través del Test de la Mirada (Baron-Cohen, Wheelwright, \& Hill, 2001) y de la adaptación al español del Interpersonal Reactivity Index (PérezAlbéniz, de Paúl, Etxeberría, Montes, \& Torres, 2003).

\section{Procedimiento}

Una vez realizado el contacto con el colegio participante, se convocó a los padres de los niños que tenían un diagnóstico de TND, realizado por un profesional, a una reunión en la que se explicaron los objetivos de la investigación, se aclararon las dudas $\mathrm{y}$ se firmaron los consentimientos informados. En segundo lugar, se tuvo un encuentro con cada estudiante para explicarles la intención del estudio y obtener, de aquellos interesados en participar voluntariamente, la firma del asentimiento informado. En tercer lugar, se comenzó el proceso de evaluación con el K-BIT para seleccionar la muestra de acuerdo con los criterios de inclusión. Finalmente, se aplicaron a los estudiantes las pruebas descritas anteriormente para la exploración de las funciones ejecutivas y la cognición social. Para cada una de ellas se calcularon las siguientes medidas.

\section{Funciones ejecutivas}

1) Test de Ejecución Auditiva Continua

- EAC_a = Número de respuestas correctas.

- EAC_b = Número de omisiones.

- EAC_c $=$ Número de comisiones.

2) Test de la Figura Compleja de Rey Osterrieth - FCR_c = Puntuación en la tarea de copia. -FCR_Tc = Tiempo empleado en llevar a cabo la copia.

- FCR_r = Puntuación en la tarea de recobro. -FCR_Tr=Tiempo empleado en llevar a caboel recobro.

3) Test de Símbolos y Dígitos

- SDMT_o = Número de ítems correctos en la ejecución verbal.

- SDMT_e = Número de ítems correctos en la ejecución escrita.

4) Test de Clasificación de Tarjetas de Wisconsin

- WCST_c = Número de categorías identificadas correctamente.

-WCST_a = Número total de aciertos. 
- WCST_e = Número total de errores.

- WCST_ep=Número total de errores perseverativos.

- WCST_enp = Número total de errores no perseverativos.

-WCST_f=Número total de fallas para mantener la actitud.

5) Test de Rastreo

-TMT_A = Tiempo empleado para la ejecución de la parte $A$.

- TMT_B = Tiempo empleado para la ejecución de la parte $\mathrm{B}$.

6) Test de asociación controlada de palabras

- FVS = Puntuación obtenida en la tarea semántica.

- FVF = Puntuación obtenida en la tarea fonológica.

7) Curva de Memoria Verbal

- CMV_a = Número de palabras recordadas en el primer intento.

-CMV_b= Número total de palabras recordadas en la ejecución.

- CMV_c = Número de intento máximo al que llegó el estudiante cuando logró recordar todas las palabras.

- CMV_d = Número de palabras recordadas en el intento 10 .

8) Test de Colores y Palabras de Stroop

- STROOP $p=$ Número de respuestas correctas en la condición palabra.

-STROOP_c = Número de respuestas correctas en la condición color.

-STROOP_pc = Número de respuestas correctas en la condición palabra-color.

\section{Cognición social}

1) Test de la Mirada

- TdM = Número de errores

2) Interpersonal Reactivity Index

- IR = Puntuación obtenida en la Adaptación al español.

Una vez que se llevó a cabo el proceso de evaluación, los datos obtenidos fueron ingresados a una base de datos en Excel y exportados para el análisis del coeficiente de correlación Rho de Spearman por medio del programa estadístico SPSS (versión 21).

\section{Resultados}

En la Tabla 1 se describen los datos demográficos de los participantes evaluados. La mayoría de los niños fue de sexo masculino, sus edades en el momento de ser evaluados osciló entre los 7 y 11 años. En relación con su grado escolar, cursaron entre 2 y 6 años (sólo uno corresponde a escolaridad secundaria).
Tabla 1. Características sociodemográficas de los 9 estudiantes evaluados

\begin{tabular}{cccccc}
\hline Edad & $\mathrm{n}$ & Sexo & $\mathrm{n}$ & $\begin{array}{c}\text { Años } \\
\text { Escolaridad }\end{array}$ & $\mathrm{n}$ \\
\hline 7 & 1 & Masculino & 6 & 2 & 2 \\
8 & 2 & Femenino & 3 & 3 & 2 \\
9 & 3 & & & 4 & 3 \\
10 & 0 & & & 5 & 1 \\
11 & 3 & & & 6 & 1 \\
\hline
\end{tabular}

En la Tabla 2 se aportan los descriptivos estadísticos de las puntuaciones obtenidas por los niños evaluados en cada una de las pruebas destacando las puntuaciones mínimas, máximas, la media y la desviación estándar.

En la Tabla 3 y 4 se pueden observar valores tanto positivos como negativos en las correlaciones, indicando aumento o disminución, de acuerdo a las relaciones presentadas entre las pruebas y entre las dimensiones o subpruebas de cada prueba en general.

Para la prueba IR, se observan relaciones muy altas e inversamente y directamente proporcionales con EAC_a y EAC_b respectivamente. De otra parte, con FCR_c, y FCR_Tc, se presentan igualmente relaciones moderadas pero directamente proporcionales. Asimismo, se encontraron relaciones medias e inversamente proporcionales, con TMT_B, lo mismo ocurre con FVF y CMV_a. Con los resultados de $\mathrm{CMV}$ _b, y con $\mathrm{CMV}$ _c, se presenta una relación moderada pero directamente proporcional.

Finalmente, los errores obtenidos en el Test de la mirada tuvieron una relación negativa con CMV_c y directamente proporcional con CMV_d.

\section{Discusión}

El objetivo del presente estudio fue la exploración de la relación entre cognición social y funciones ejecutivas en niños con TND. A continuación se analizan las relaciones significativas encontradas. Si bien los resultados que se reportan no pueden ser generalizados a la población en general, pueden orientar la exploración de estas relaciones en futuras investigaciones. Las mismas contribuirán a ayudar al entendimiento de esta problemática y encaminar las acciones de intervención para la prevención, habilitación y rehabilitación que hacen diversos profesionales en el ámbito escolar.

En primer lugar se destaca que en los participantes evaluados se encontró que la 
Tabla 2. Resultados obtenidos por los participantes en cada una de las pruebas evaluadas

\begin{tabular}{lcccc}
\hline \multicolumn{1}{c}{ Pruebas } & Mínimo & Máximo & Media & dt \\
\hline K-BIT & 83,00 & 115,00 & 97,89 & 11,16 \\
EAC_a & 8,00 & 15,00 & 11,89 & 2,26 \\
EAC_b & 1,00 & 8,00 & 3,89 & 2,32 \\
EAC_c & 0,00 & 7,00 & 1,22 & 2,54 \\
FCR_c & 11,00 & 33,00 & 24,39 & 8,59 \\
FCR_Tc & 93,00 & 350,00 & 207,44 & 80,66 \\
FCR_r &, 50 & 24,00 & 10,00 & 7,84 \\
FCR_Tr & 8,00 & 488,00 & 105,78 & 147,88 \\
SDMT_o & 21,00 & 49,00 & 32,44 & 9,80 \\
SDMT_e & 13,00 & 60,00 & 31,00 & 14,53 \\
WCST_c & 1,00 & 6,00 & 3,67 & 1,66 \\
WCST_a & 68,00 & 93,00 & 78,78 & 9,92 \\
WCST_e & 25,00 & 60,00 & 47,33 & 12,70 \\
WCST_ep & 6,00 & 39,00 & 22,33 & 10,63 \\
WCST_enp & 11,00 & 37,00 & 24,33 & 7,23 \\
WCST_f & 0,00 & 4,00 & 2,22 & 1,30 \\
TMT_A & 69,00 & 300,00 & 148,44 & 76,41 \\
TMT_B & 165,00 & 360,00 & 207,33 & 59,66 \\
FVS & 8,00 & 32,00 & 21,78 & 8,11 \\
FVF & 13,00 & 66,00 & 23,33 & 16,50 \\
CMV_a & 2,00 & 7,00 & 4,11 & 1,69 \\
CMV_b & 2,00 & 10,00 & 8,78 & 2,64 \\
CMV_c & 4,00 & 10,00 & 7,00 & 2,98 \\
CMV_d & 8 & 10 & 9,00 & 1,00 \\
STROOP_p & 53,00 & 74,00 & 63,33 & 7,68 \\
STROOP_c & 31,00 & 57,00 & 47,89 & 10,08 \\
STROOP_pc & 16,00 & 28,00 & 22,00 & 4,58 \\
TdM & 8,00 & 16,00 & 12,33 & 2,65 \\
IR & 25,00 & 49,00 & 33,11 & 7,52 \\
\hline
\end{tabular}

Nota: Curva Memoria Verbal: CMV_a = Número de palabras recordadas en el primer intento. CMV_b = Número total de palabras recordadas. CMV_c = Número de intento máximo al que llegó el estudiante cuando logró recordar todas las palabras. CMV_d = Número de palabras recordadas en el intento 10. Test de ejecución auditiva continua: EAC_a $=$ Número de respuestas correctas. EAC_b $=$ Número de omisiones. EAC_c $=$ Número de comisiones. Test de Figura Compleja de Rey Osterrieth: FCR_c $=$ Puntuación en la tarea de copia. FCR_Tc $=$ Tiempo empleado en llevar a cabo la copia. FCR_r = Puntuación en la tarea de recobro. FCR_Tr = Tiempo empleado en llevar a cabo el recobro. Test de Asociación Controlada de palabras: FVS = Puntuación obtenida en la tarea semántica. FVF = Puntuación obtenida en la tarea fonológica. Interpersonal Reactivity Index: IR = Puntuación obtenida en la Adaptación al español. Test de Símbolos y Dígitos: SDMT_o = Número de ítems correctos en la ejecución verbal. SDMT_e = Número de ítems correctos en la ejecución escrita. Test de Colores y Palabras de Stroop: STROOP_p = Número de respuestas correctas en la condición palabra. STROOP_c = Número de respuestas correctas en la condición color. STROOP_pc = Número de respuestas correctas en la condición palabra-color. Test de la Mirada: $\mathbf{T d M}=$ Número de errores. TMT_A $=$ Test de Rastreo: Tiempo empleado para la ejecución de la parte A. TMT_B = Tiempo empleado para la ejecución de la parte B. Test de Clasificación de Tarjetas de Wisonsin: WCST_c $=$ Número de categorías identificadas correctamente. WCST_a = Número total de aciertos. WCST_e = Número total de errores. WCST_ep = Número total de errores perseverativos. WCST_enp = Número total de errores no perseverativos. WCST_f $=$ Número total de fallas para mantener la actitud.

empatía, explorada por la Adaptación al español del Interpersonal Reactivity Index (IR), se relaciona con varias habilidades de las funciones ejecutivas.

Diversos autores han identificado, entre otros, compromisos en la empatía en niños con TND (Barry et al., 2013; Peña-Olvera, \& Palacios-Cruz, 2011;), denominados actualmente como callo emocional (Barry et al., 2013; Kolko \& Pardini, 2010; Masi et al., 2014; Pardini \& Fite, 2010;
Peña-Olvera \& Palacios-Cruz, 2011; Waschbusch et al., 2007).

\section{Empatía y control inhibitorio}

Muñoz et al. (2004) encontraron que en los primeros niveles de educación media se encontraron dificultades inhibitorias importantes en niños con TND. En el presente estudio, se observó una relación inversamente proporcional con el control de impulsos (evaluado por el Test 
Tabla 3. Correlaciones entre pruebas de Cognición Social y Función Ejecutiva de 9 niños que presentan TND

\begin{tabular}{|c|c|c|c|c|c|c|c|c|c|c|c|c|}
\hline $\begin{array}{l}\text { Coeficiente } \\
\text { correlación } \\
\text { Rho de } \\
\text { Spearman }\end{array}$ & $\sum_{0}$ & $\cong$ & $\underbrace{\pi}_{\text {Uు }}$ & 号 & 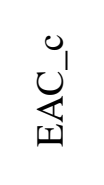 & 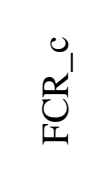 & 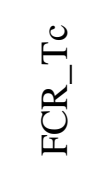 & ب̂ & 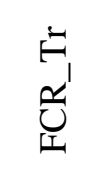 & $\sum_{\substack{n \\
\vdots}}^{0}$ & $\sum_{n}^{\infty}$ & $\begin{array}{l}0 \\
v_{1} \\
\tilde{v}_{3} \\
3\end{array}$ \\
\hline TdM & 1,000 &,- 100 &,- 245 &, 120 & ,332 &,- 101 &,- 025 & ,021 & ,113 & ,092 & ,008 &,- 090 \\
\hline IR & & 1,000 &,- 727 & ,741 & 091 & ,644 & ,500 &,- 142 &,- 276 & , 150 & 0,000 &,- 188 \\
\hline EAC_a & & & 1,000 &,- 983 &,- 059 &,- 416 &,- 180 & ,206 & ,064 &,- 154 &, 120 & ,068 \\
\hline EAC_b & & & & 1,000 &,- 093 & ,346 & ,085 &,- 256 &,- 098 & ,060 &,- 247 & ,391 \\
\hline EAC_c & & & & & 1,000 & ,504 & 639 & 023 &,- 034 & ,274 & ,707 &, 325 \\
\hline FCR_c & & & & & & 1,000 & ,778 & ,340 & ,269 & 661 & 695 &,- 367 \\
\hline FCR_Tc & & & & & & & 1,000 & ,485 & ,117 & ,350 & ,567 &,- 059 \\
\hline FCR_r & & & & & & & & 1,000 & ,798 & ,460 & ,326 & ,386 \\
\hline FCR_Tr & & & & & & & & & 1,000 & ,485 & 268 & 299 \\
\hline SDMT_o & & & & & & & & & & 1,000 & ,767 & ,794 \\
\hline SDMT_e & & & & & & & & & & & 1,000 &, 627 \\
\hline WCST_c & & & & & & & & & & & & 1.000 \\
\hline
\end{tabular}

Tabla 4. (continuación) Correlaciones entre pruebas de Cognición Social y Función Ejecutiva de 9 niños que presentan TND

\begin{tabular}{|c|c|c|c|c|c|c|c|c|c|c|c|c|}
\hline $\begin{array}{l}\text { Coeficiente } \\
\text { correlación Rho } \\
\text { de Spearman }\end{array}$ & $\sum_{\theta}$ & $\cong$ & $\sum_{i}^{\infty}$ & $\sum_{i}^{\infty}$ & $\sum_{I}^{L}$ & $\sum_{0}^{\pi}$ & $\sum_{0}^{2}$ & $\sum_{i}^{u}$ & $\sum_{j}^{z_{1}}$ & 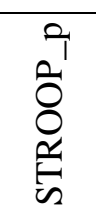 & 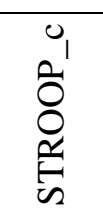 & 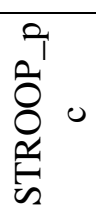 \\
\hline TdM & 1,00 &,- 100 & ,119 &,- 273 &,- 097 & , 094 &,- 030 &,- 504 & ,656 &,- 287 & ,050 &,- 186 \\
\hline IR & & 1,00 &,- 509 & ,126 &,- 504 &,- 553 & ,644 &,- 451 & , 198 &,- 017 & 025 & , 176 \\
\hline EAC_a & & & ,330 &,- 009 & ,586 & ,031 &,- 792 & ,271 &,- 467 &,- 134 & ,013 &,- 306 \\
\hline EAC_b & & &,- 251 &,- 013 &,- 665 &,- 096 & ,819 & -,205 & ,329 & ,064 &,- 124 & ,258 \\
\hline EAC_c & & &,- 650 & ,275 & 483 & , 280 &,- 230 &,- 434 & ,366 & ,334 & ,596 & , 380 \\
\hline FCR_c & & &,- 740 & , 143 &,- 156 &,- 162 & 313 &,- 551 & ,328 & ,527 & 298 & 679 \\
\hline FCR_Tc & & &,- 695 & ,075 & 269 &,- 136 &,- 030 &,- 325 & 248 & 437 & 427 & 202 \\
\hline FCR_r & & &,- 128 &,- 525 & , 110 &,- 026 &,- 338 &,- 050 & ,070 & ,422 &,- 109 &, 025 \\
\hline FCR_Tr & & &,- 060 &,- 668 &,- 198 & , 145 &,- 283 & 138 &,- 005 & 380 &,- 387 & ,325 \\
\hline SDMT_o & & &,- 542 & ,059 &,- 034 & ,017 & 168 &,- 601 & ,545 & 647 & 276 & 672 \\
\hline SDMT_e & & &,- 610 & 377 & ,420 & 264 &,- 099 &,- 651 & 416 & 681 & ,636 & 706 \\
\hline WCST_c & & &, 035 &,- 090 & ,112 & ,026 &,- 102 &,- 374 & ,229 & ,453 & , 159 & ,259 \\
\hline WCST_a & & & 103 &,- 298 &,- 017 &,- 165 &,- 423 &,- 128 &,- 040 & ,085 &,- 179 & 214 \\
\hline WCST_e & & &,- 017 & ,230 & ,026 & ,338 & ,312 & ,128 & , 040 &,- 128 & , 136 &,- 154 \\
\hline WCST_ep & & & ,120 & 468 & ,055 & ,502 & ,429 & , 114 &,- 020 & , 008 & 262 & , 051 \\
\hline WCST_enp & & &,- 119 &,- 420 & ,025 & -,282 &,- 462 & 626 &,- 437 &,- 511 &,- 433 &,- 527 \\
\hline WCST_f & & & , 071 &,- 479 &,- 723 &,- 447 & ,036 & 493 &,- 421 &,- 556 &,- 808 &,- 048 \\
\hline TMT_B & & & 1,00 &,- 128 &,- 137 & ,104 &,- 030 & ,138 &,- 171 &,- 547 &,- 289 &,- 538 \\
\hline FVS & & & & 1,00 & ,422 & ,316 & ,403 &,- 466 & ,283 & ,278 & ,824 & ,316 \\
\hline FVF & & & & & 1,00 & ,489 &,- 524 & ,051 & ,025 & ,369 & ,667 &,- 106 \\
\hline CMV_a & & & & & & 1,00 &,- 015 & ,065 & ,248 & ,549 & ,466 & ,343 \\
\hline CMV_b & & & & & & & 1,00 &,- 655 & ,476 & , 135 & ,194 & ,320 \\
\hline CMV_c & & & & & & & & 1,00 &,- 860 &,- 266 &,- 626 &,- 317 \\
\hline CMV_d & & & & & & & & & 1,00 & ,295 & ,577 & ,250 \\
\hline STROOP_p & & & & & & & & & & 1,00 & 498 & 682 \\
\hline STROOP_c & & & & & & & & & & & 1,00 & ,287 \\
\hline STROOP_pc & & & & & & & & & & & & 1,00 \\
\hline
\end{tabular}


de ejecución auditiva continua) que requieren tareas visuales en las cuales los niños deben inhibir respuestas no deseadas, en las que juega un papel importante la atención selectiva y la atención sostenida. En otras palabras, en los niños evaluados cuando existen dificultades en el control inhibitorio mediado por la atención, aumenta la preocupación empática. Cuantas más omisiones (saltos atencionales) tuvieron estos niños, mayores puntuaciones obtuvieron en la preocupación empática. Lo anterior, pone de manifiesto una hipótesis interesante para explorar en futuros estudios, tendientes a responder al siguiente interrogante ¿Será que los procesos voluntarios como la atención selectiva en estos niños interfieren con la capacidad de establecer empatía?

\section{Empatía y organización/planeación visoespacial}

El Test de la Figura Compleja de Rey, en la exploración ejecutiva, permite evaluar la planeación y organización que hacen las personas para llevar a cabo una tarea visoespacial y es sensible a lesiones en el Lóbulo temporal izquierdo. En este caso, se encontró que cuando estos niños tenían menores puntuaciones en esta prueba tanto en la ejecución como en el tiempo empleado (dificultades en la organización y planeación visoespacial), menos empatía tenían.

\section{Empatía y secuenciación}

Se encontró una relación inversamente proporcional entre la medición de la empatía y la parte B del Test de rastreo lo cual indica que a menor secuenciación, mayor empatía.

\section{Empatía y conciencia fonológica}

El Test de asociación controlada de palabras es sensible a lesiones en el hemisferio izquierdo específicamente en regiones dorsolaterales. En este caso, los resultados obtenidos sugieren que a menor conciencia fonológica, mayor empatía en estos niños.

\section{Empatía y estrategias ejecutivas en memoria de trabajo y memoria a corto plazo verbal}

$\mathrm{Si}$ bien la memoria de trabajo no es propiamente una habilidad de las funciones ejecutivas, la estrategia requerida para la retención de información necesaria para llevar a cabo una tarea específica sí lo es. Los resultados obtenidos en la Curva de memoria verbal sugieren que un compromiso en dichas estrategias aumenta en estos niños la empatía. En cuanto a las estrategias para la memoria a corto plazo, se encontró una relación directamente proporcional con la empatía, es decir, al parecer compromisos en las estrategias para la memoria a corto plazo afectan la empatía en los niños evaluados. Lo anterior, se confirma con la relación inversamente proporcional encontrada con la medición CMV_c, ya que a menor número de intentos requeridos para recordar todas las palabras (mejores estrategias ejecutivas) mayor empatía.

En segundo lugar, se destacan las relaciones encontradas entre la teoría de la mente evaluada por el Test de la mirada y habilidades de las funciones ejecutivas en los niños evaluados con TND. Diversos autores han identificado problemas en la reacción emocional de estos niños como consecuencia de dificultades en el procesamiento de información emocional propia y de otros (Ison-Zintilini, \& Morelato, 2008; Masi et al., 2014).

\section{Teoría de la mente y estrategias de aprendizaje}

Las estrategias de aprendizaje fueron evaluadas por la Curva de memoria verbal. Entre los resultados obtenidos en esta prueba, se encontró que cuando menos intentos requirieron los niños evaluados para aprender todas las palabras de la prueba, mejor empatía evidenciaban. De igual manera, se puso de manifiesto que a mayor aprendizaje, mayor empatía. Lo anterior sugiere que compromisos en estrategias de aprendizaje afectan la empatía de estos niños.

Los resultados obtenidos en la presente investigación concuerdan con los hallazgos de Matthys et al. (2012) quienes encuentran que los procesos de aprendizaje en esto trastorno se ven afectado por disfunciones neurocognitivas dentro de las cuales están las funciones ejecutivas. Estos autores habían identificado compromisos en los siguientes procesos: aprendizaje, control inhibitorio, resolución de problemas, dificultades atencionales, falta de flexibilidad cognitiva y toma de decisiones. Todo lo anterior compromete la capacidad de estos niños de aprender a optimizar su comportamiento en ambientes cambiantes.

Los compromisos en las funciones ejecutivas alteran la cognición social de estos niños, por lo cual, la capacidad de regulación emocional y estrategias adecuadas de planeación y ejecución de conductas tienen como consecuencia las respuestas agresivas y el déficit de respuestas prosociales (Ison-Zintilini \& Morelato, 2008).

Como se ha mencionado anteriormente, los resultados obtenidos en la presente investigación no permiten la generalización de los hallazgos a la población estudiada. Sin embargo, podrían orientar futuras investigaciones. 


\section{Referencias}

American Psychiatric Association (2003). DSMIV-TR: Manual Diagnóstico y Estadístico de los Trastornos Mentales (1a. ed.). España: Masson, S.A.

American Psychiatric Association. (2013). Diagnostic and statistical manual of mental disorders (5th ed.). Washington, DC: Author.

Ardila, A., \& Rosselli, M. (1992). Neuropsicología clínica. Medellín: Prensa Creativa.

Baron-Cohen, S., Wheelwright, S. \& Hill, J. (2001). The 'Reading the mind in the eyes' test revised version: A study with normal adults, and adults with Asperger Syndrome or HighFunctioning autism. Journal of Child Psychology and Psychiatry, 42, 241-252.

Barry, C. T., Golmaryami, F. N., Rivera-Hudson, N., \& Frick, P. J. (2013). Evidence-based assessment of conduct disorder: Current considerations and preparation for DSM-5. Professional Psychology: Research and Practice, 44(1), 56.

Corsi, E., Barrera, P., Flores, C., Perivancich, X., $\&$ Guerra, C. (2009). Efectos de un programa combinado de técnicas de modificación conductual para la disminución de la conducta disruptiva y el aumento de la conducta prosocial en escolares chilenos. Acta Colombiana de Psicología, 12(1), 67-76

Coy, K., Speltz, M. L., DeKlyen, M., \& Jones, K. (2001). Social--cognitive processes in preschool boys with and without oppositional defiant disorder. Journal of Abnormal Child Psychology, 29(2), 107-119

de Ancos, E. T., \& Ascaso, L. E. (2011). Sex differences in oppositional defiant disorder. Psicothema, 23(4), 666-671.

Ezpeleta, L., De la Osa, N., Granero, R., \& Trepat, E. (2014). Functional impairment associated with symptoms of oppositional defiant disorder in preschool and early school boys and girls from the general population. Anales de Psicología, 30(2), 395-402.

Flory, K., Milich, R., Lynam, D., Leukefeld, C., \& Clayton, R. (2003). Relation between childhood disruptive behavior disorders and substance use and dependence symptoms in young adulthood: individuals with symptoms of attention-deficit/hyperactivity disorder and conduct disorder are uniquely at risk. Psychology of Addictive Behaviors, Vol 17(2), 151-158.

Forehand, R., Jones, D.J., \& Parent, J. (2013). Behavioral parenting interventions for child disruptive behaviors and anxiety: What's different and what's the same, Clinical Psychology Review, 33(1), 133-145.

Golden, C.J. (2001). Stroop: Test de Colores y Palabras. Madrid: TEA Ediciones, S.A.

Guaita, V.L. (2007). Aspectos emocionales de la comunicación en niños en riesgo por pobreza extrema. Revista Iberoamericana de Evaluación - e Avaliação Psicológica, 24(2), 177-192.

Heaton, R.K., Chelune, G.J. Talley, J.L., Kay, G.G., \& Curtiss (2001). Test de Clasificación de Tarjetas de Wisconsin manual (2da edición). Madrid: TEA Ediciones, S.A.

Ison, M.S. (2004). Características familiares y habilidades sociocognitivas en niños con conductas disruptivas. Revista Latinoamericana de Psicología, 36(2):257268

Ison-Zintilini, M. S., \& Morelato, G. S. (2008). Habilidades socio-cognitivas en niños con conductas disruptivas y víctimas de maltrato. Univ. Psychol, 7(2): 357-367.

Kaufman, A.S., \& Kaufman, N.L. (1997). K-BIT. Test breve de inteligencia de Kaufman. Madrid: TEA

Kolko, D. J., \& Pardini, D. A. (2010). ODD dimensions, ADHD, and callous-unemotional traits as predictors of treatment response in children with disruptive behavior disorders. Journal of Abnormal Psychology, 119(4), 713-725. doi:10.1037/a0020910

Lezak, M. D. (1995). Neuropsychological assessment. (3a. Ed.). New York: Oxford University.

Lezak, M.D., Howieson, D.D., \& Loring, D.W. (2004). Neuropsychological Assessment (4th $E d)$. New York: Oxford University Press.

McKinney, C., \& Morse, M. (2012). Assessment of Disruptive Behavior Disorders: Tools and Recommendations. Professional Psychology, 
Research \& Practice, 43(6), 641-649. doi: 10.1037/a0027324

Masi, G., Milone, A., Pisano, S., Lenzi, F., Muratori, P., Gemo, I., \& Vicari, S. (2014). Emotional reactivity in referred youth with disruptive behavior disorders: The role of the callous-unemotional traits. Psychiatry Research, 220(1/2), 426-432. doi:10.1016/j.psychres.2014.07.035.

Matthys, W., \& Cuperus, J. M. (1999). Deficient social problem-solving in boys with ODD/CD, with ADHD, and with both disorders. Journal of the American Academy of Child \& Adolescent Psychiatry, 38(3), 311321.

Matthys, W., Vanderschuren, L., Schutter, D., \& Lochman, J. (2012). Impaired neurocognitive functions affect social learning processes in oppositional defiant disorder and conduct disorder: Implications for interventions. Clinical Child \& Family Psychology Review, 15(3), 234-246. doi:10.1007/s10567-0120118-7.

Muñoz, J. M., Carreras, M., \& Braza, P. (2004). Aproximación al estudio de las actitudes y estrategias de pensamiento social y su relación con los comportamientos disruptivos en el aula en la educación secundaria. Anales de Psicología, 20(1), 81-91.

Pardini, D. A., \& Fite, P. J. (2010). Symptoms of conduct disorder, oppositional defiant disorder, attention-deficit/hyperactivity disorder, and callous-unemotional traits as unique predictors of psychosocial maladjustment in boys: Advancing an evidence base for DSM-V. Journal of the American Academy of Child \& Adolescent Psychiatry, 49(11), 1134-1144. doi:10.1016/j.jaac.2010.07.010.

Peña-Olvera, F. 1., \& Palacios-Cruz, L. (2011). Trastornos de la conducta disruptiva en la infancia y la adolescencia: diagnóstico y tratamiento. Salud Mental, 34(5), 421-427.

Pérez-Albéniz, A., de Paúl, J., Etxeberría, J., Montes, M.P., \& Torres, E. (2003). Adaptación de Interpersonal Reactivity Index (IRI) al español. Psicothema, 15(2), 267-272.

Pino, M., \& García, M.T. (2007). Concepto, tipos y etiología de las conductas disruptivas en un centro de Educación Secundaria y Bachillerato desde la perspectiva del profesorado. Revista de Pedagogía, 28(81), 111-134.

Rabadán, J.A., \& Giménez, A.M. (2012). Detección e intervención en el aula de los trastornos de conducta. Educación XX1, 15(2), 185-212.

Reitan, R.M., \& Wolfson, D. (1993). The Halstead-Reitan Neuropsychological Test Battery: Theory and clinical interpretation (2 $2^{\text {nd }}$. Edition). Tucson, AZ: Neuropsychology Press.

Restrepo, J.C., \& Molina, D.A (2011). Neuropsicología y funciones ejecutivas. En Corporación Universitaria Lasallista (Comp., 2011). Desarrollo y Transversalidad de la Serie Lasallista de investigación y ciencia, (pp. 49-66). Medellín: Artes y Letras.

Rey, A. (2003). Test de Copia y Reproducción de Memoria de Figuras Complejas. Madrid: TEA Ediciones, S.A.

Roa, M. L., \& Del Barrio, M. V. (2003). Estructura de personalidad materna: $\mathrm{Su}$ implicación en los problemas infantiles. Revista Iberoamericana de Evaluación - e Avaliação Psicológica, 16(2), 53-69.

Robles, Z., \& Romero, E. (2011). Programas de entrenamiento para padres de niños con problemas de conducta: Una revisión de su eficacia. Anales de psicología, 27, 86-101

Román, F.N., Rojas, G., Román, N.R., Iturry, M., Blanco, R., Leis, A., Bartoloni, L., Allegri, R.F., \& Grupo de trabajo Programa Argencog (2012). Baremos del Test de la Mirada en español en adultos normales de Buenos Aires. Revista Neuropsicología Latinoamericana, 4(3), 1-5.

Sliminng, E., Barrera, P., Flores, C., Perivancich, X., \& Guerra, C. (2009). Efectos de un programa combinado de técnicas de modificación conductual para la disminución de la conducta disruptiva y el aumento de la conducta prosocial en escolares chilenos. Acta Colombiana de Psicología, 12(1), 67-76.

Smith, A. (2002). SDMT: Test de Símbolos y Dígitos. Madrid: TEA Ediciones, S.A.

van Lier, P. C., Huizink, A., \& Vuijk, P. (2011). The role of friends' disruptive behavior in the 
development of children's tobacco experimentation: Results from a preventive intervention study. Journal of Abnormal Child Psychology, 39(1), 45-57. doi:10.1007/s10802-010-9446-6.

Waschbusch, D. A., Walsh, T. M., Andrade, B. F., King, S., \& Carrey, N. J. (2007). Social problem solving, conduct problems, and callous-unemotional traits in children. Child Psychiatry \& Human Development, 37(4), 293-305. doi:10.1007/s10578-006-0033-6. 